\title{
Expression of two members of the Wnt family during mouse development-restricted temporal and spatial patterns in the developing neural tube
}

\author{
Henk Roelink and Roel Nusse \\ Howard Hughes Medical Institute and Department of Developmental Biology, Beckman Center, Stanford University, \\ Stanford, California 94305 USA
}

\begin{abstract}
The Wnt gene family encodes a group of cysteine-rich proteins implicated in intercellular signaling during several stages of vertebrate development. This family includes Wnt-1 and Wnt-3, both discovered as activated oncogenes in mouse mammary tumors. Here we describe the molecular cloning of an additional member of the Wnt family, called Wnt-3A, and the spatial and temporal expression pattern of this gene as well as that of its close relative Wnt-3. The putative amino acid sequences of both proteins are almost $90 \%$ identical, but in situ hybridization to mouse embryo sections showed highly restricted patterns of expression of Wnt-3 and Wnt-3A, largely in separate areas in the developing nervous system. In the spinal cord Wnt-3 was expressed at low levels in the alar laminae and in the ventral horns, whereas Wnt-3A expression was confined to the roof plate. In the developing brain Wnt-3 was expressed broadly across the dorsal portion of the neural tube with a rostral boundary of expression at the diencephalon. In contrast, Wnt-3A was expressed in a narrow region very close to the midline; expression extended into the bifurcating telencephalon, in a highly localized fashion. Both Wnt-3 and Wnt-3A were expressed in the ectoderm, and Wnt-3A was also expressed in the periumbilical mesenchyme. Characteristic expression patterns of these two closely related genes suggest that Wnt-3 and Wnt-3A play distinct roles in cell-cell signaling during morphogenesis of the developing neural tube.
\end{abstract}

[Key Words: Wnt family of genes; developmental expression; in situ hybridization]

Received November 15, 1990; revised version accepted December 27, 1990.

The classical description of the developing mouse embryo has provided a framework by which to study mammalian development at a molecular level. Among several different strategies for identifying genes that control mammalian embryogenesis, two approaches have proven particularly rewarding in recent years. Of the many genes identified by virtue of their ability to promote tumor formation when mutated or activated, several have been implicated in the control of the fundamental steps of cellular differentiation (Jakobovits et al. 1986; Wilkinson et al. 1989|, leading to the suggestion that these proto-oncogenes have normal functions during development (Nusse 1988). Second, a steadily increasing number of mammalian genes are being identified because of their sequence similarity to genes known previously to control developmental decisions in Drosophila and/or Caenorhabditis elegans. In many cases, ontogenic function, as well as sequence similarity, appears to be evolutionarily conserved. Identification and isolation of the mouse Wnt gene family is a successful example of both approaches.
The Wnt-1 (int-1) (Nusse and Varmus 1982) and Wnt-3 (Roelink et al. 1990) genes were first recognized as protooncogenes due to their activation in mouse mammary tumors as a consequence of nearby insertion of mouse mammary tumor virus (MMTV) proviral DNA. It was subsequently shown that the Drosophila homolog of Wnt- 1 is wingless, a segment polarity gene implicated in a cascade of cell-cell communication events that establish developmental compartments in the early embryo (Rijsewijk et al. 1987). Additional members of the Wnt family are $W n t-2$ (irp), detected by its proximity to a CpG island in the vicinity of the human CFTR gene (Wainwright et al. 1988), and at least six other members identified by enzymatic amplification using degenerate primers (Gavin et al. 1990).

Biochemical studies of the mouse Wnt-1 protein show that it is glycosylated and secreted (Papkoff et al. 1987) but remains tightly associated with the cell surface (Papkoff and Schryver 1990) and/or extracellular matrix (Bradley and Brown 1990). These observations are supported by genetic and biochemical analysis of the wingless gene 
product, which acts in a cell nonautonomous fashion (Morata and Lawrence 1977) over relatively short distances of several cell diameters (Van den Heuvel et al. 1989). Sequence comparison among pairs of Wnt gene family members reveals $\sim 45 \%$ similarity at the amino acid residue level, as well as complete conservation of 22 cysteine residues, a potential amino-terminal-linked glycosylation site, and the presence of a signal peptide. It therefore seems likely that all members of the Wnt gene family are involved in aspects of cell-cell communication.

Do different members of the Wnt gene family serve redundant or exclusive roles during mammalian embryogenesis? Functional redundancy may be a general hallmark of developmental gene families and is supported by the multiplicity and sometimes overlapping expression pattern of mouse Hox genes (Graham et al. 1989) and the minimal phenotypic consequences of inactivating the mouse en-2 gene (A. Joyner, pers. comm.). Although the mouse Wnt-l gene is expressed along the entire spinal cord, its inactivation produces defects in development of the midbrain and the cerebellum only (McMahon and Bradley 1990; Thomas and Capecchi 1990). To address these issues and to better understand the role of Wnt genes in mouse development, we have characterized the expression patterns of Wnt-3 and a new closely related gene, Wnt-3A. In contrast with Wnt-1, we find that Wnt3 and Wnt-3A are both expressed in the developing forebrain. Many areas are highly delimited and only partially overlap with each other, which suggests that Wnt-1, $W n t-3$, and $W n t-3 \mathrm{~A}$ have unique roles in morphogenesis of the developing neural tube.

\section{Results \\ Cloning of Wnt-3A cDNA clones}

During the isolation of Wnt-3 cDNA clones from a day 8.5 mouse embryo cDNA library (the gift of B. Hogan) with a genomic probe (pBG87) containing the third and part of the fourth exon of the Wnt-3 gene (Roelink et al. 1990), strongly and weakly hybridizing plaques were identified under high-stringency conditions. The strongly hybridizing plaques represented cDNA clones of Wnt-3 itself. Five weakly hybridizing plaques were isolated and sequenced. All five cDNA inserts represented transcripts derived from the same gene, called Wnt-3A. One cDNA clone contained a complete putative open reading frame, which was colinear with the amino acid sequences of the other Wnt proteins (Fig. 1).

The putative Wnt-3A translation product is $91 \%$ identical to the Wnt-3 protein sequence, disregarding the nonconserved amino-terminal signal peptide. The cysteine pattern in both proteins is identical. Many of the remaining differences between the Wnt-3 and Wnt-3A protein sequences were substitutions of similar amino acid residues. The Wnt-3A protein had an amino-terminal signal sequence and two possible $\mathrm{N}$ linked glycosylation sites, one of which is conserved in all members of the Wnt family. The conceptual primary translation product of Wnt-3A contains 352 amino acid residues, with a predicted molecular mass of 39,257 daltons. As the cDNA sequences in the $3^{\prime}$ - and $5^{\prime}$ untranslated parts do not show any significant similarity, we used 3'-untranslated fragments as gene-specific probes specifically to detect expression of $W n t-3$ and Wnt-3A.

\section{Expression of Wnt-3 and Wnt-3A during development}

By RNA blot analysis we found a Wnt-3A transcript of $3.1 \mathrm{~kb}$ in adult lung but not in liver, heart, spleen, kidney, testis, skeletal muscle, mammary gland, brain, and uterus (data not shown). In embryos, the first detectable expression of Wnt-3 and Wnt-3A was observed around day 10 of embryogenesis. As the Wnt-3 and Wnt-3A cDNA clones were isolated from a day 8.5 mouse cDNA library, we expected low levels of expression earlier than day 10, but we have been unable to detect significant expression, either by RNA blot analysis or by in situ hybridization.

Serial transverse and sagittal sections of day 10.5-12.5 embryos were used to determine the exact areas of Wnt-3 and Wnt-3A expression by in situ hybridization. We performed control sense strand hybridizations to sections adjacent to those presented and never observed any signal above background.

\section{Wnt -3 and Wnt-3A expression in the day 10.5 embryo}

The neural tube of a day 10.5 embryo consists of a number of morphologically distinct regions. From caudal to rostral, these regions are the spinal cord, the myelencephalon (hindbrain), the mesencephalon (midbrain), the diencephalon, and the two bilateral chambers of the telencephalon. Because of the strong curvature of the embryos, transverse sections can pass twice through the neural tube.

We found Wnt- 3 expression in the dorsal portion of all regions of the neural tube except in the telencephalon and in the hindbrain. In the hindbrain, Wnt-3 was expressed in the junction between the neuroepithelium and the thin roof of ependymal cells (superior membrane) (Fig. 2A,Cl. More posterior and anterior, these regions merge; expression of Wnt-3 encircled the complete superior membrane of the myelencoel. Wnt-3 was broadly expressed in the dorsal portion of the midbrain and diencephalon. The rostral boundary of Wnt-3 expression lies in the telencephalic flexure of the prosencephalon, which marks the division between the developing diencephalon and telencephalon (Fig. 2A). The Wnt-3 expression in the neural tube was continuous in length.

Like Wnt-3, the Wnt-3A gene was expressed in the dorsal portion of the neural tube but within a much smaller region. In the spinal cord, Wnt-3A was expressed only in the developing roof plate. Anteriorly, this localized midline expression bifurcates to accommodate the superior membrane of the rhombencephalon; in this area, expression of Wnt-3 and Wnt-3A was coincident, 
Wnt-3A TACGGTCAAG GCAGAGGGCC CAGCGCCACT GCAGCCGCGC CACCTCCCAG GGCCGGGCCA GCCCAGGCGT CCGCGCTCTC GGGGTGGACT CCCCCCCGCTG

200 CGCGCTCAAG CCGGCGATGG CTCCTCTCGG ATACCTCTTA GTGCTCTGCA GCCTGAAGCA GGCTCTGGGC AGCTACCCGA TCTGGTGGTC CTTGGCTGTG

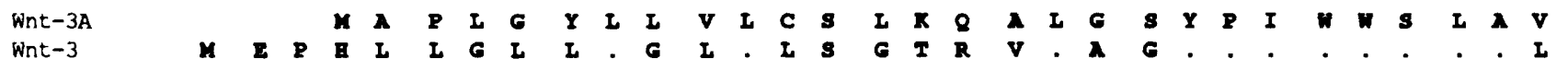
WhT-3 GGACCCCAGT ACTCCTCTCT GAGCACTCAG CCCATTCTCT GTGCCAGCAT CCCAGGCCTG GTACCGAAGC AGCTGCGCTT CTGCAGGAAC TACGTGGAGA

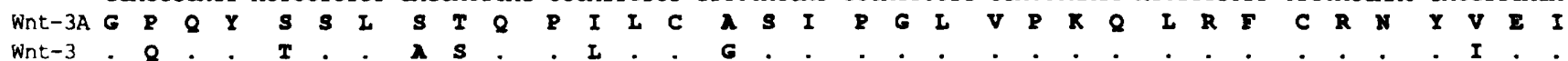
Wnt-3. $2 \cdot \cdot \cdot 100$ TCATGCCCAG CGTGGCTGAG GGTGTCAAAG CGGGCATCCA GGAGTGCCAG CACCAGTTCC GAGGCCGGCG TTGGAACTGC ACCACCGTCA GCAACAGCCT

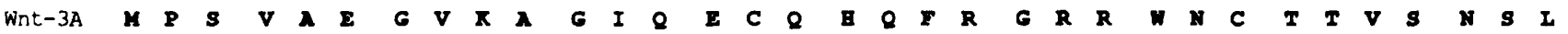

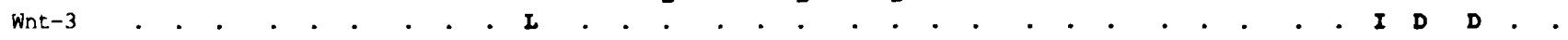

GGCCATCTTT GGCCCTGTTC TGGACAAAGC CACCCGGGAG TCAGCCTTTG TCCATGCCAT CGCCTCCGCT GGAGTAGCTT TCGCAGTGAC ACGCTCCTGT

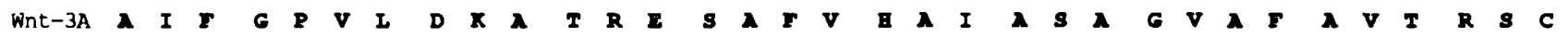
Wnt-

GCAGAGGGAT CAGCTGCTAT CTGTGGGTGC AGCAGCCGCC TCCAGGGCTC CCCAGGCGAG GGCTGGAAGT GGGGCGGCTG TAGTGAGGAC ATTGAATTTG $\begin{array}{lllllllllllllllllllllllllllllllllllll}\text { Wnt-3A } & \mathbf{A} & \mathbf{E} & \mathbf{G} & \mathbf{S} & \mathbf{A} & \mathbf{A} & \mathbf{I} & \mathbf{C} & \mathbf{G} & \mathbf{C} & \mathbf{S} & \mathbf{S} & \mathbf{R} & \mathbf{L} & \mathbf{Q} & \mathbf{G} & \mathbf{S} & \mathbf{P} & \mathbf{G} & \mathbf{E} & \mathbf{G} & \mathbf{W} & \mathbf{K} & \mathbf{W} & \mathbf{G} & \mathbf{G} & \mathbf{C} & \mathbf{S} & \mathbf{E} & \mathbf{D} & \mathbf{I} & \mathbf{E} & \mathbf{F} & \mathbf{G} \\ \text { Wnt-3 } & \cdot & \cdot & \cdot & \mathbf{T} & \mathbf{S} & \mathbf{T} & \cdot & \cdot & \cdot & \cdot & \mathbf{D} & \cdot & \mathbf{B} & \mathbf{B} & \mathbf{X} & \cdot & \mathbf{P} & \cdot & \cdot & \cdot & \cdot & \cdot & \cdot & \cdot & \cdot & \cdot & \cdot & \cdot & \cdot & \cdot & \mathbf{A} & \mathbf{D} & \cdot & \cdot\end{array}$

GAGGAATGGT CTCTCGGGA TTTGCCGATG CCAGGGAGAA CCGGCCGGAT GCCCGCTCTG CCATGAACCG TCACAACAAT GAGGCTGGGC GCCAGGCCAT

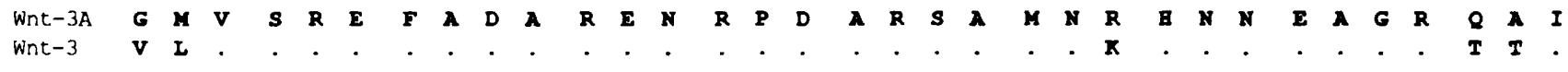

CGCCAGTCAC ATGCACCTCA AGTGCAAATG CCACGGGCTA TCTGGCAGCT GTGAAGTGAA GACCTGCTGG TGGTCGCAGC CGGACTTCCG CACCATCGGG

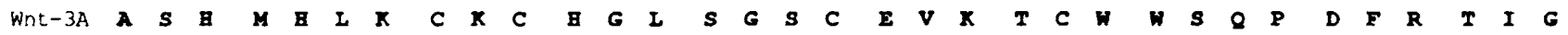

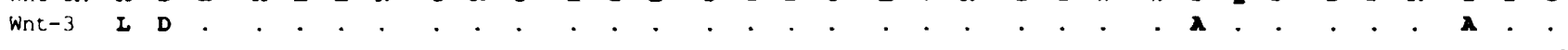

GATTTCCTCA AGGACAAGTA TGACAGTGCC TCGGAGATGg TGGTAGAGAA ACACCGAGAG TCTCGTGGCT GGgTGGAGAC CCTGAGGCCA CGTTACACGT

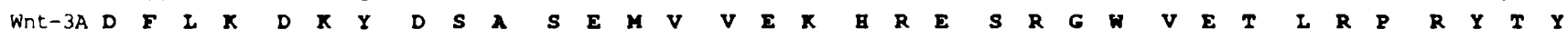

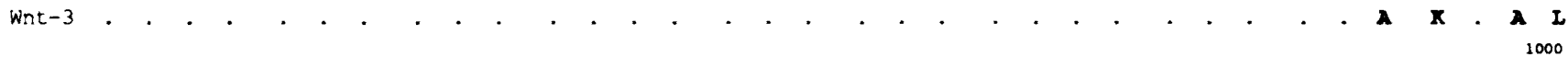
ACTTCAAGGT GCCGACAGAA CGCGACCTGG TCTACTACGA GGCCTCACCC AACTTCTGCG AACCTAACCC CGAAACCGGC TCCTTCGGGA CGCGTGACCG

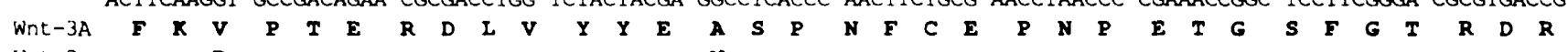

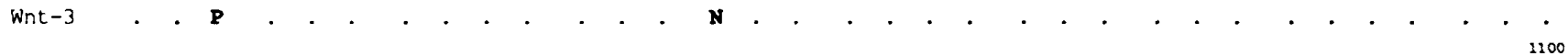

CACCTGCAAT GTGAGCTCGC ATGGCATAGA TGGGTGCGAC CTGTTGTGCT GCGGGCGCGG GCATAACGCG CGCACTGAGC GACGGAGGGA GAAATGCCAC

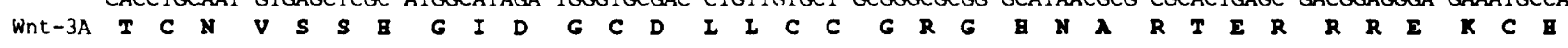

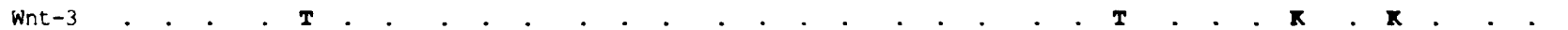

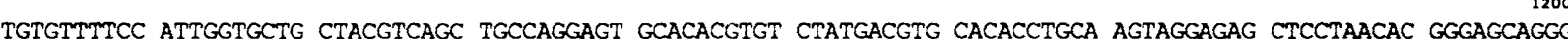

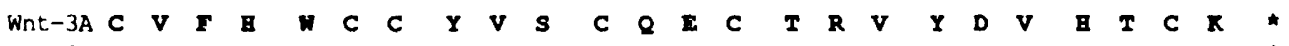
Wnt-3 . . . . . . . . . . . . . . I . I . . . . . . . * *

Figure 1. Nucleotide sequence of the first 1200 bases of clone $15 \mathrm{~A}$ representing $W n t-3 \mathrm{~A}$ transcripts. The putative amino acid sequence of the Wnt-3 protein is given in single-letter code. The asterisk (*) indicates the stop codon. Below the Wnt-3A amino acid sequence, the identical residues (dots) and nonidentical residues (single-letter code) of the Wnt-3 amino acid sequence are shown.

although Wnt-3A expression was confined to a smaller area (Fig. $2 \mathrm{~B}, \mathrm{D}$ ). Rostral to the myelencephalon, Wnt-3A was again expressed in the dorsal midline in the position of the developing cerebellum. Moving rostral still farther, midline expression of Wnt-3A again bifurcated at the telencephalic flexure and was expressed in small areas of the bilateral developing telencephalic bulbs. (Fig. 2D).

Outside the neural tube, Wnt-3 was expressed in the dorsal ectoderm, the branchial arches, and the limb buds (Fig. 2A,C), and Wnt-3A was expressed in the mesenchyme tissue surrounding the umbilical veins (Fig. 2B).

\section{Wnt-3 and Wnt-3A expression in the day 11.5 embryo}

At day 11.5, the telencephalic bulbs have expanded and the telencephalic flexure has deepened. The developing dorsal thalamus is still a relatively thin layer of cells, although some distinct expanding areas are evident. On midsagittal and parasagittal sections, the expression patterns of Wnt-3 and Wnt-3A were generally similar to that seen at 10.5 days. The $W n t-3$ gene was broadly expressed in the dorsal portion of the neural tube with a rostral boundary in the dorsal thalamus. The anterior edge of the boundary was very sharp, just caudal of the telencephalic flexure (Fig. 3I), but the lateral edges were indistinct (Fig. 3G,I). In the hindbrain, expression continued to surround the roof of the myelencephalon (Fig. $3 \mathrm{~A}, \mathrm{D}, \mathrm{G}, \mathrm{J}, \mathrm{M})$.

Within the telencephalon, Wnt-3A was highly expressed in areas just dorsolateral of the telencephalic flexure (Fig. $3 \mathrm{H}, \mathrm{K}, \mathrm{N})$. On midsagittal sections, Wnt-3A expression was also seen in a narrow band along the dorsal midline (Fig. 3B) that extended nearly to the rostral 


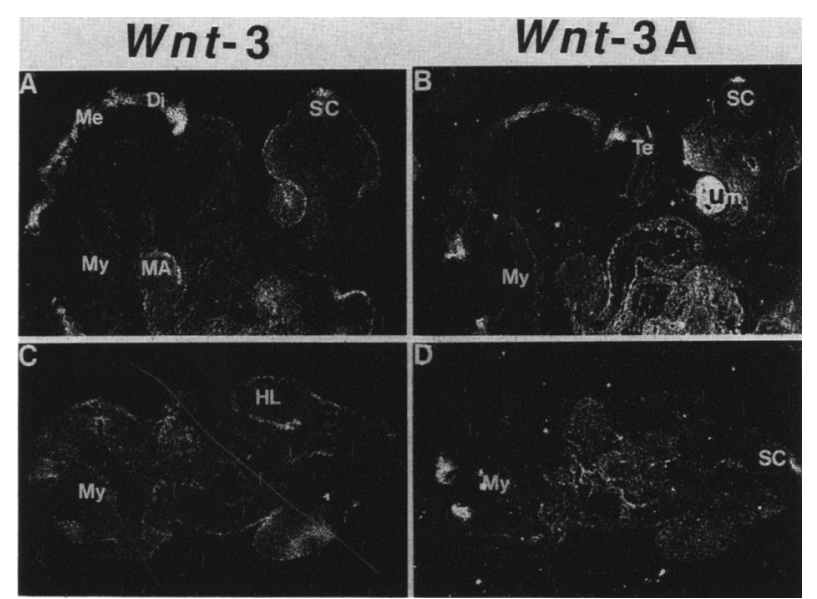

Figure 2. Dark-field photomicrographs of day 10.5 mouse embryo sagittal $(A$ and $B)$ and transverse $\{C$ and $D \mid$ sections, hybridized with $W n t-3(A$ and $C)$ and $W n t-3 A(B$ and $D)$ antisense RNA probes. White areas and lines represent expression; grayness of the tissue is caused by aspecific light scattering. (Te) Telencephalon; (Di) diencephalon; (Me) mesencephalon; (My) myelencephalon; (SC) spinal cord; (Um) umbilicus; (MA) mandibular arch; (HL) hind limb.

tip of the neuraxis. Within the diencephalon, Wnt-3A was expressed in a small area at the boundary between the dorsal and ventral thalamus (Fig. $3 \mathrm{~K}$ ). Wnt-3A expression continued to surround the myelencephalic roof but appeared much closer to the midline than Wnt-3 expression (Fig. 3B,E,H,K,N).

\section{Wnt-3 and Wnt-3A expression in the day 12.5 embryo}

At day 12.5, the same regions in the neural tube described for day 10.5 embryos can still be identified. The dorsal thalamus has grown significantly, and expression is easier to interpret because of the larger size of the embryos.

In the spinal cord, cells have differentiated further, and more functional areas can be distinguished. Transverse sections of the spinal cord showed a broad area of Wnt-3 expression in the alar laminae of the spinal cord, similar to that seen in day 10.5 embryos. In addition, Wnt-3 was expressed in the ventral horns, the area where the cell bodies of differentiating motor neurons are located (Fig. $5 \mathrm{~A})$. Expression in the ventral horns was also detected in a few consecutive sagittal sections (Fig. 5C). In the developing brain, Wnt-3 was strongly expressed in the dorsal thalamus (Fig. 4A,C,E). Sagittal sections showed a rostral boundary of Wnt-3 expression just behind the telencephalic flexure, in addition to a low level of expression in the dorsal portion of the mesencephalon and the cerebellum (Fig. 4A). In the hindbrain around the myelencephalic roof, Wnt-3 expression has decreased sharply compared with that seen in younger embryos.

Expression of Wnt-3A in the spinal cord in day 12.5 embryos was confined to a triangle-shaped group of cells that form the presumptive roof plate (Fig. 5B). In the brain, expression was observed in the dorsal midline up to the diencephalon (Fig. 4D), except in the hindbrain where, as at earlier times, a band of midline expression bifurcated to accommodate the diencephalic roof (Fig. $5 \mathrm{D})$. In the diencephalon, the line of Wnt-3A expression bifurcated again (Fig. 4F) to extend into the medial walls of the telencephalon (Fig. 4D,F). Within the diencephalon, a small area of Wnt-3A expression was observed in both sagittal and transverse sections that marked the boundary between the dorsal and ventral thalamus (Fig. $4 B, D \mid$, but expression was never extended into ventral structures. Wnt-3A expression proved highest around the dorsal portion of the telencephalic flexure (Fig. 4B). On transverse sections, it was clear that Wnt-3A expression surrounded the lateral and posterior choroid plexus (Figs. 4F and 5D).

At day 12.5, outside of the nervous system Wnt-3 and Wnt-3A were expressed generally throughout the ectoderm. Relatively low levels were observed in the ectoderm covering the backs; higher levels were found in the ectoderm covering the limb buds and in the caudal position of the ectoderm covering the mandibular arch (Fig. $5 \mathrm{E}, \mathrm{F})$. The epithelium lining the forming oral cavity did not express $W n t-3$ and $W n t-3 \mathrm{~A}$. Within the mandibular arch, Wnt-3 and Wnt-3A expression patterns were very similar, although higher for Wnt-3. In addition, Wnt-3A expression extended slightly further into the oral cavity.

\section{Discussion}

\section{Expression of Wnt-3 and Wnt-3A in the neural tube}

A number of gene families have been implicated in establishing compartments in the brain, based on their ex pression patterns: They are the Hox (Graham et al. 1989), Pax (Chowdhury et al. 1988), and En (Davis and Joyner 1988) gene families. Most of these genes, however, are expressed in the ventral portion of the neural tube and have their most anterior boundary of expression in the spinal cord or in the hindbrain (Kessel and Gruss 1990). This contrasts with Wnt-3 and Wnt-3A, which are expressed in the dorsal portion of the neural tube and have their most anterior boundaries of expression in the diencephalon and the telencephalon, suggesting that no in teraction of these genes and/or their products occurs.

Wnt-3A is expressed very strongly in the dorsal portion of the boundary between the diencephalon and both cerebral hemispheres. This area is part of the archicortex, a phylogenetically older system than the neocortex, and includes structures involved in emotional and affective behavior such as the hippocampus and the amygdala. Division of the prosencephalon and diencephalon is a relatively recent event in vertebrate evolution, and it seems possible that duplication of an ancestral gene giving rise to the Wnt-3 and Wnt-3A genes occurred coincident with this division. If so, both genes may serve similar processes from both morphogenetic and phylogenetic perspectives. On the basis of our results, this process probably involves cell-cell signaling within the dorsal thalamus and dorsal archicortex. 


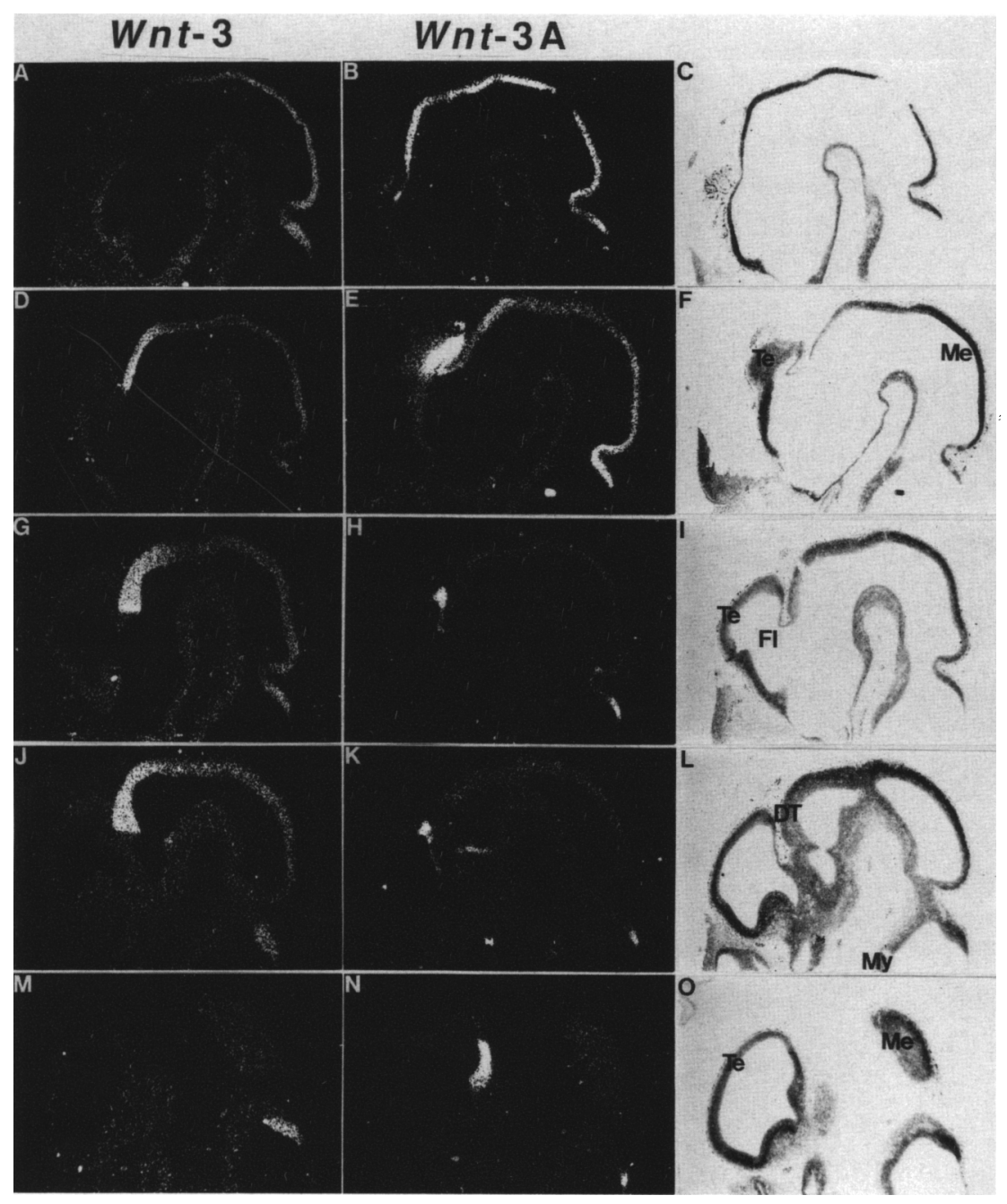

Figure 3. Dark-field photomicrographs of day 11.5 mouse embryo sagittal sections, hybridized with Wnt-3 $(A, D, G, J$, and $M)$ and Wnt-3A $(B, E, H, K$, and $N)$ antisense RNA probes. $C, F, I, L$, and $O$ are brightfield photographs of the sections hybridized with Wnt-3A. From top to bottom, midsagittal to lateral sagittal sections are shown. (Te) Telencephalon; (DT) dorsal thalamus (Me) mesencephalon; (FI) foramen interventriculare; $(\mathrm{My})$ myelencephalon. All photographs were taken at the same magnification.
In the spinal cord, Wnt-3 is expressed in the ventral horns concomitant with motor neuron differentiation (Wentworth 1984). We speculate that the Wnt-3 protein might serve as a chemotropic axon-guidance molecule. Alternatively, the Wnt-3 protein might support growth and differentiation of neuron or glia cells. Despite the expression of the Wnt-3 gene in the dorsal portion of the spinal cord, we have never observed any Wnt-3 expression in cells or tissue derived from the neural crest.

We are struck by the expression of Wnt-3A along the dorsal midline of the entire neuraxis, sparing only the myelencephalic roof. Possibly, Wnt-3A may play a role in establishing the dorsal midline and/or serving as a reference point for further dorsoventral differentiation. In the chick spinal cord, both the roof plate and the floor plate elaborate signals necessary for the proper dorsoventral differentiation (Wagner et al. 1990). The presumed properties of the Wnt proteins as short-range cellcell signaling molecules could well be the source of such differentiation signals and could easily act in the brain as well as the spinal cord. Furthermore, it seems possible that additional, as yet undiscovered, Wnt genes may be the source of floor plate signals.
These questions can be addressed most conveniently by manipulating the site of Wnt-3A expression, either via transgenic animals or by transplantation experiments coupled with in situ hybridization. Both approaches are feasible, although the transplantation studies are best addressed in the chick.

\section{Expression of Wnt-3 and Wnt-3A outside the neural tube}

Within the mesodermally derived tissues, only Wnt-3A is expressed in the mesenchyme surrounding the umbilical veins in the day 10.5 embryo. This area is coincident with that described for Wnt-2 expression (McMahon and McMahon 1989|. Within the ectoderm, Wnt-3 and Wnt$3 \mathrm{~A}$ are expressed most highly in the caudal portion of the mandibular arches. Ferguson (1988) has speculated that the ectoderm plays an important role in establishing the caudal/rostral identity of the branchial arches by generating inductive signals. The products of Wnt-3 and Wnt$3 \mathrm{~A}$ might help to establish the differences between the rostral (bone and teeth forming) and caudal side of the mandibular arch. The onset of Wnt-3 expression in the 


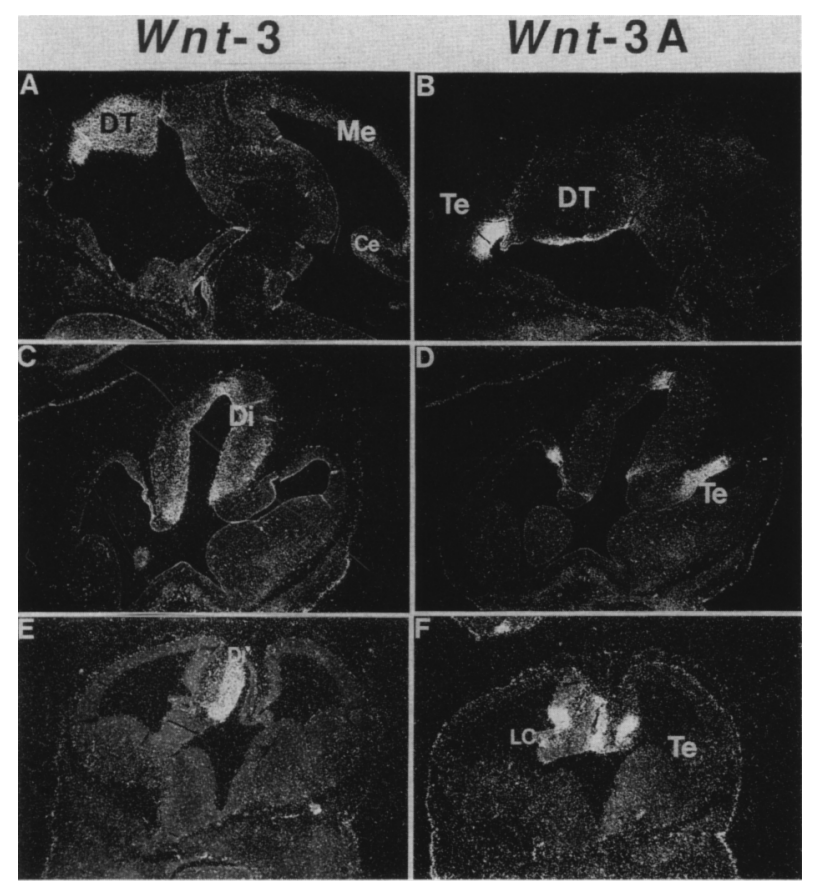

Figure 4. Dark-field photomicrographs of transverse $(C, D, E$, and $F)$ and sagittal $(A$ and $B)$ sections of the brain of day 12.5 mouse embryo hybridized with Wnt-3 $(A, C$, and $E)$ and Wnt-3A $(B, D$, and $F)$ antisense RNA probes. White areas represent expression, grayness of the tissue is caused by light scattering in the tissues. (Te) Telencephalon; (DT) dorsal thalamus; (Di) diencephalon; (Me) mesencephalon; $(\mathrm{Ce})$ cerebellum; (LC) lateral choroid plexus. All photographs were taken at the same magnification.

branchial arch ectoderm is much earlier and stays at much higher levels than Wnt-3A expression.

\section{The Wnt family of genes}

The Wnt-1 gene now appears to be part of a large gene family that consists of at least 10 members in the mouse. Apart from the novel member, Wnt-3A, reported here, some six other related genes have been identified recently through polymerase chain reactions (PCRs) with degenerate primers (Gavin et al. 1990). From the expression pattern of some of these genes it has been inferred that they play important roles during differentiation of several organs in midgestation embryos, particularly in the developing nervous system. For Wnt-1, the prototype member of this family, such an essential function has recently been demonstrated by generating mouse strains that lack a functional Wnt-1 allele. These homozygous insertional null mutations of the Wnt-1 gene affect the formation of those areas in the midbrain where a dorsoventral ring of Wnt-1-expressing cells is detected as well as adjacent cerebellar structures (McMahon and Bradley 1990; Thomas and Capecchi 1990). Compared with the pattern described for Wnt-1 expression, Wnt-3A expression completely overlaps that of Wnt-1 in several notable areas (Wilkinson et al. 1987a). In particular, Wnt-
1 expression in the roof plate and surrounding the myelencephalic roof is identical to Wnt-3A expression. If Wnt-1 and Wnt-3A are functionally redundant proteins, this may explain why mice with an inactivated Wnt-1 gene apparently have a normal spinal cord and hindbrain.

The expression patterns shown here suggest that the Wnt- 3 and Wnt-3A genes, like Wnt-1, might also play a role in the brain morphogenesis but at other areas. In particular, the rostral boundaries of Wnt-3 and Wnt-3A expression are anterior to Wnt-1 expression, which suggests that Wnt-3 and Wnt-3A may control morphogenesis of areas that are most highly developed in humans.

\section{Materials and methods}

\section{Tissue preparation}

Timed pregnant BALB/C and CD-1 mice were identified by the appearance of the vaginal plug. Because matings took place around midnight, the age of the embryos was considered to be 0.5 day on the day of appearance of the vaginal plug. Embryos were removed at the desired ages and fixed overnight in $4 \%$ paraformaldehyde dissolved in PBS. The next day, the embryos were dried through an ethanol series and stored in $80 \%$ ethanol. Ethanol-dried, xylene-treated embryos were embedded in wax,

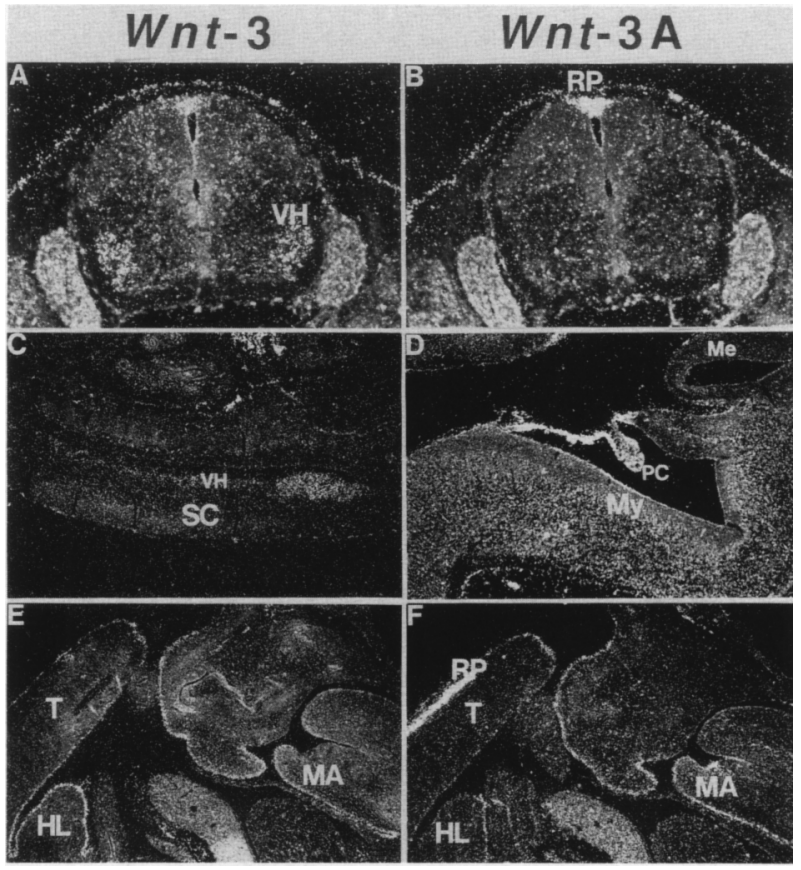

Figure 5. Dark-field microphotographs of transverse and sagittal sections of day 12.5 mouse embryo, hybridized with Wnt-3 $(A, C$, and $E)$ and $W n t-3 A(B, D$, and $F)$ antisense RNA probes. $\mid A$ and $B)$ Transverse sections through the spinal cord; $|C\rangle$ a parasagittal section through the anterior part of the spinal cord; $(D)$ a parasagittal section through the hindbrain and the cerebellum. $(E$ and $F)$ Parasagittal sections through the ventral part of the embryo. (Me) Mesencephalon; $(\mathrm{RP})$ roof plate; $(\mathrm{PC})$ posterior choroid plexus; (SC) spinal cord; (VH) ventral horn; (My) myelencephalon; (MA) mandibular arch; $(\mathrm{T})$ tail; (HL) hind limb bud. 
and ribbons of $6-\mu \mathrm{m}$ sections were cut on a microtome. Sections were transferred to 3 -aminopropyltriethoxysilane-coated (Sigma) glass slides and stored desiccated at $4^{\circ} \mathrm{C}$.

Organs for RNA preparation were dissected out, immediately frozen in liquid nitrogen, and stored at $-70^{\circ} \mathrm{C}$.

\section{Isolation and subcloning of the Wnt-3A cDNA clones}

Wnt-3A cDNAs were identified by hybridization of $5 \times 10^{6}$ recombinant phage of a day 8.5 mouse embryo cDNA library with probe pBG87 (Roelink et al. 1990) under high-stringency conditions $\left(65^{\circ} \mathrm{C}, 3 \times \mathrm{SSC}\right)$. Subsequent washes were performed in $0.1 \times \mathrm{SSC}$ at $65^{\circ} \mathrm{C}$. pBG87 contains the third and part of the fourth exon of the Wnt-3 gene. Phage DNA was isolated according to established procedures (Sambrook et al. 1989), and the cDNA inserts were released from the phage arms with EcoRI. The isolated cDNA fragments were subcloned in the EcoRI site of $\mathrm{pGEM}-3 \mathrm{Zf}|+|$.

\section{Wnt-3A cDNA sequencing}

Wnt-3A clones $9 \mathrm{~A}$ and $15 \mathrm{~A}$, the longest fragments obtained, were sequenced by using primers specific for the phage $\mathrm{T} 7$ and SP6 promoters as internal primers. All sequence reactions were performed on denatured double-stranded templates by using Sequenase version 2 according to the manufacturer's instructions. Both strands of clones $9 \mathrm{~A}$ and $15 \mathrm{~A}$ were sequenced. Other clones were identified on partial sequences. No other clones extended $5^{\prime}$ or $3^{\prime}$ over clone $9 \mathrm{~A}$ or $15 \mathrm{~A}$. The Wnt-3A cDNA sequence including all available sequence 3 ' of the coding domain will appear in the EMBL and DDBJ Nucleotide Sequence Databases under accession number X56842.

\section{In situ hybridization}

In situ hybridizations were performed according to an established protocol (Wilkinson et al. 1987b). Wnt-3 and Wnt-3A probes were derived from noncoding and nonconserved cDNA fragments. The Wnt-3 probe is a $1.2-\mathrm{kb}$ SalI-EcoRI fragment cloned in pGEM-3Zf $\mid+1$. The Wnt-3A probe is a $750-b p$ EcoRIBgllI fragment also cloned in pGEM-3Zf $+1 .\left[{ }^{35}\right.$ S UTP-labeled transcripts of the Wnt-3 and Wnt-3A clones were generated using established procedures (Melton et al. 1984). Antisense Wnt3 transcripts were generated using T7 RNA polymerase on Sall linearized template; antisense transcripts of the Wnt-3A clone were generated using SP6 polymerase on an EcoRI linearized template. Probes were degraded to an average length of $100 \mathrm{bp}$ by controlled alkaline lysis (Cox et al. 1984). Sections were hybridized at $59^{\circ} \mathrm{C}$ for $6-16 \mathrm{hr}$ and washed according to Wilkinson et al. (1987b) at $60^{\circ} \mathrm{C}$. Slides were dipped in Kodak NBT-2 emulsion, dried, and exposed at $4^{\circ} \mathrm{C}$ for $4-10$ days. Exposure times were judged on overnight exposure to XAR2 film (Kodak). Slides were developed in D19 developer (Kodak), and the emulsion was fixed in $30 \%$ sodium thiosulfate. Sections were stained with methyl green, mounted, and examined and photographed using Kodak Tech Pan film, under dark-field illumination.

\section{Acknowledgments}

We thank Liesbeth van Deemter and Els Wagenaar for their contribution to the cloning and sequencing of the Wnt-3A cDNA clones. We thank Dr. B. Hogan for providing the mouse embryo cDNA library and Dr. S. McConnell for her help in interpreting the in situ data. We thank Dr. G. Barsh for helpful discussions and Drs. G. Barsh and S. Hughes for their comments on the manuscript.
The publication costs of this article were defrayed in part by payment of page charges. This article must therefore be hereby marked "advertisement" in accordance with 18 USC section 1734 solely to indicate this fact.

\section{References}

Bradley, R.S. and A.M. Brown. 1990. The proto-oncogene int-1 encodes a secreted protein associated with the extracellular matrix. EMBO I. 9: 1569-1575.

Chowdhury, K., G. Dressler, G. Breier, U. Deutsch, and P. Gruss. 1988. The primary structure of the murine multifinger gene $\mathrm{mKr} 2$ and its specific expression in developing and adult neurons. EMBO I. 7: 1345-1353.

Cox, K. H., L.M. DeLeon, L.M. Angerer, and R.C. Angerer. 1984. Detection of mRNAs in sea urchin embryos by in situ hybridization using asymetric RNA probe. Dev. Biol. 101: 485502.

Davis, C.A. and A.L. Joyner. 1988. Expression patterns of the homeo box-containing genes En-I and En-2 and the protooncogene int-1 diverge during mouse development. Genes $\Theta$ Dev. 2: 1736-1744.

Ferguson, M.W. 1988. Palate development. Development (suppl.) 103: 41-60.

Gavin, B.J., J.A. McMahon, and A.P. McMahon. 1990. Expression of multiple novel Wnt-1/int-1-related genes during fetal and adult mouse development. Genes \& Dev. 4: 2319-2332.

Graham, A., N. Papalopulu, and R. Krumlauf. 1989. The murine and Drosophila homeobox gene complexes have common features of organization and expression. Cell 57: 367-378.

Jakobovits, A., G.M. Shackleford, H.E. Varmus, and G.R. Martin. 1986. Two proto-oncogenes implicated in mammary carcinogenesis, int -1 and int -2 , are independently regulated during mouse development. Proc. Natl. Acad. Sci. 83: 78067810 .

Kessel, M. and P. Gruss. 1990. Murine developmental control genes. Science 249: 374-379.

McMahon, A.P. and A. Bradley. 1990. The Wnt-1 (int-1) protocncogene is required for development of a large region of the mouse brain. Cell 62: 1073-1082.

McMahon, J.A. and A.P. McMahon. 1989. Nucleotide sequence, chromosomal localization and developmental expression of the mouse int-1-related gene. Development 107: 643-650.

Melton, D.A., P.A. Krieg, M.R. Rebagliati, T. Maniatis, K. Zinn, and M.R. Green. 1984. Efficient in vitro synthesis of biologically active RNA and RNA hybridization probes from plasmids containing a bacteriophage SP6 promoter. Nucleic ACids Res. 12: 7035-7056.

Morata, G. and P.A. Lawrence. 1977. The development of wingless, a homeotic mutation in Drosophila. Dev. Biol. 99: 261264.

Nusse, R. 1988. The int genes in mammary tumorigenesis and in normal development. Trends Genet. 4: 291-295.

Nusse, R. and H.E. Varmus. 1982. Many tumors induced by the mouse mammary tumor virus contain a provirus integrated in the same region of the host genome. Cell 31: 99-109.

Papkoff, J. and B. Schryver. 1990. Secreted int-1 protein is associated with the cell surface. Mol. Cell. Biol. 10: 2723-2730.

Papkoff, J., A.M. Brown, and H.E. Varmus. 1987. The int-1 proto-oncogene products are glycoproteins that appear to enter the secretory pathway. Mol. Cell. Biol. 7: 3978-3984.

Rijsewijk, F., M. Schuermann, E. Wagenaar, P. Parren, D. Weigel, and R. Nusse. 1987. The Drosophila homolog of the mouse mammary oncogene int- $I$ is identical to the segment polarity gerie wingless. Cell 50: 649-657. 
Roelink, H., E. Wagenaar, S. Lopes da Silva, and R. Nusse. 1990. Wnt-3, a gene activated by proviral insertion in mouse mammary tumors, is homologous to int-1/Wnt-1 and normally expressed in mouse embryos and adult brain. Proc. Natl. Acad. Sci. 87: 4519-4523.

Sambrook, J., E.F. Fritsch, and T. Maniatis. 1989. Molecular cloning: A laboratory manual, 2nd ed. Cold Spring Harbor Laboratory Press, Cold Spring Harbor, New York.

Shackleford, G.M. and H.E. Varmus. 1987. Expression of the proto-oncogene int-1 is restricted to postmeiotic male germ cells and the neural tube of mid-gestational embryos. Cell 50: 89-95.

Thomas, K.R. and M.R. Capecchi. 1990. Targeted disruption of the murine int- 1 proto-oncogene resulting in severe abnormalities in midbrain and cerebellar development. Nature 346: 847-850.

Van den Heuvel, M., R. Nusse, P. Johnston, and P.A. Lawrence. 1989. Distribution of the wingless gene product in Drosophila embryos: A protein involved in cell-cell communication. Cell 59: 739-749.

Wagner, M., C. Thaller, T. Jessell, and G. Eichele. 1990. Polarizing activity and retinoid synthesis in the floor plate of the neural tube. Nature 345: 819-822.

Wainwright, B.J., P.J. Scambler, P. Stanier, E.K. Watson, G. Bell, C. Wicking, X. Estivill, M. Courtney, A. Boue, P.S. Pedersen, R. Williamson and M. Farrall. 1988. Isolation of a human gene with protein sequence similarity to human and murine int- 1 and the Drosophila segment polarity mutant wingless. EMBO I. 7: 1743-1748.

Wentworth, L.E. 1984. The development of the cervical spinal cord of the mouse embryo. I. A Golgi analysis of ventral root neuron differentiation. /. Comp. Neurol. 222: 81-95.

Wilkinson, D.G., J.A. Bailes, and A.P. McMahon. 1987a. Expression of the proto-oncogene int-1 is restricted to specific neural cells in the developing mouse embryo. Cell 50: 79-88.

Wilkinson, D.G., J.A. Bailes, J.E. Champion, and A.P. McMahon. 1987b. A molecular analysis of mouse development from 8 to 10 days post coitum detects changes only in embryonic globin expression. Development 99: 493-500.

Wilkinson, D.G., S. Bhatt, and A.P. McMahon. 1989. Expression pattern of the FGF-related proto-oncogene int-2 suggests multiple roles in fetal development. Development 105: 131136. 


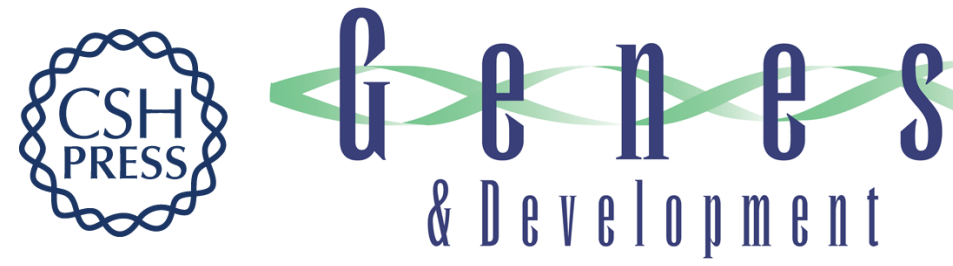

\section{Expression of two members of the Wnt family during mouse development--restricted temporal and spatial patterns in the developing neural tube.}

H Roelink and R Nusse

Genes Dev. 1991, 5:

Access the most recent version at doi:10.1101/gad.5.3.381

References This article cites 28 articles, 11 of which can be accessed free at: http://genesdev.cshlp.org/content/5/3/381.full.html\#ref-list-1

License

Email Alerting Receive free email alerts when new articles cite this article - sign up in the box at the top Service right corner of the article or click here.

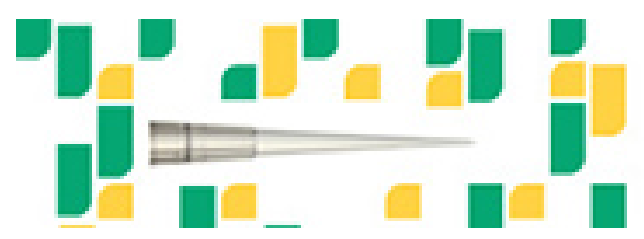

Focused on your science. 